Vol. 44 (1991) [397-404]

\title{
LOCAL MINIMA OF THE GAUSS CURVATURE OF A MINIMAL SURFACE
}

\section{ShINJI YAMASHITA}

Let $D$ be a domain in the complex $w$-plane and let $x: D \rightarrow \mathbb{R}^{3}$ be a regular minimal surface. Let $M(K)$ be the set of points $w_{0} \in D$ where the Gauss curvature $K$ attains local minima: $K\left(w_{0}\right) \leqslant K(w)$ for $\left|w-w_{0}\right|<\delta\left(w_{0}\right), \delta\left(w_{0}\right)>0$. The components of $M(K)$ are of three types: isolated points; simple analytic arcs terminating nowhere in $D$; analytic Jordan curves in $D$. Components of the third type are related to the Gauss map.

\section{INTRODUCTION AND RESULTS}

Our purpose is to study the set of parameter points where the Gauss curvature of a minimal surface in the Euclidean space $\mathbb{R}^{3}$ attains local minima. A nonconstant map $x$ from a domain $D$ in the complex $w$-plane $\mathbb{C}(w=u+i v)$ into the Euclidean space $\mathbb{R}^{3}$, in notation, $x: D \rightarrow \mathbb{R}^{3}$, is said to determine a regular minimal surface, or, simply, $x$ is a regular minimal surface defined in $D$, if the following three conditions hold:

(HA) Each component $x_{k}$ of $x=\left(x_{1}, x_{2}, x_{3}\right)$ is harmonic in $D$.

(IS) The real parameters $u$ and $v$ are isothermal in the sense that

$$
\sum_{k=1}^{3} \phi_{k}^{2} \equiv 0
$$

in $D$, where

$$
\phi_{k}=\frac{\partial x_{k}}{\partial u}-i \frac{\partial x_{k}}{\partial v}=2 \frac{\partial x_{k}}{\partial w}, \quad k=1,2,3 .
$$

(RE) The function $\sum_{k=1}^{3}\left|\phi_{k}\right|^{2}$ never vanishes in $D$.

Suppose that the surface $x$ is not contained in any plane in $\mathbb{R}^{3}$. Then $f=\phi_{1}-i \phi_{2}$ is analytic and $g=\phi_{3} / f$ is meromorphic in $D$. The Gauss map $\Gamma$ of $x$ is the map from $x$ into the unit sphere $S$ in $\mathbb{R}^{3}$ defined by

$$
\Gamma(w) \equiv \Gamma(x(w))=\left(\frac{2 \operatorname{Re} g(w)}{|g(w)|^{2}+1}, \frac{2 \operatorname{Im} g(w)}{|g(w)|^{2}+1}, \frac{|g(w)|^{2}-1}{|g(w)|^{2}+1}\right), w \in D
$$

Received 27 November 1990

Copyright Clearance Centre, Inc. Serial-fee code: 0004-9729/91 \$A2.00+0.00. 
this is the unit normal at $x(w)$ with the standard orientation, together with $\Gamma(w)=$ $(0,0,1)$, if $w$ is a pole of $g$. Then $\Gamma$ is identified with $g$ via the stereographic projection from $S$ onto $\mathbb{C} \cup\{\infty\}$. The Gauss curvature at the point $x(w)$ is then

$$
K(w)=-\left[\frac{4 g^{\#}(w)}{|f(w)|\left(1+|g(w)|^{2}\right)}\right]^{2},
$$

where the spherical derivative $h^{\#}(w)$ at $w$ of $h$ meromorphic in $D$ is defined by

$$
h^{\#}(w)= \begin{cases}\left|h^{\prime}(w)\right| /\left(1+|h(w)|^{2}\right) & \text { if } h(w) \neq \infty, \\ \left|(1 / h)^{\prime}(w)\right| & \text { if } h(w)=\infty\end{cases}
$$

Condition (RE) is valid if and only if the function

$$
|f|\left(1+|g|^{2}\right)=\sqrt{2 \sum_{k=1}^{3}\left|\phi_{k}\right|^{2}}
$$

never vanishes in $D$. Thus, if $x$ is not contained in any plane, then $K(w) \neq 0$ if and only if $g^{\#}(w) \neq 0$. This is the case if and only if $w$ is a simple pole of $g$ or $g(w) \neq \infty$ and $g^{\prime}(w) \neq 0$. Therefore, $-\infty<K \leqslant 0$ everywhere in $D$. For the basic properties of minimal surfaces, see $[\mathbf{1}, \mathbf{2}]$.

Let $M(K)$ be the set of points $w_{0} \in D$ where $K$ has local minima: $K\left(w_{0}\right) \leqslant K(w)$ for $w$ in a disk $\left\{\left|w-w_{0}\right|<\delta\right\}$ with $\delta$ depending on $K$ and $w_{0}$.

THEOREM 1. Let $x: D \rightarrow \mathbb{R}^{3}$ be a regular minimal surface contained in no plane and with nonempty $M(K)$. Then the connected components of $M(K)$ are at most countable and each component is one of the following:

(1) An isolated point.

(2) A simple analytic arc terminating nowhere in $D$.

(3) A simple closed analytic curve.

All the cases of (1), (2) and (3) actually happen; see the next section. We let $M_{1}(K), M_{2}(K)$, and $M_{3}(K)$ be the set of components of $M(K)$ of type (1), (2), and (3), respectively.

Let $D_{1}$ be a subdomain of $D$. The total curvature of the subsurface $x: D_{1} \rightarrow \mathbb{R}^{3}$ is defined by

$$
T\left(D_{1}\right)=\frac{1}{2} \iint_{D_{1}} K \cdot \sum_{k=1}^{3}\left|\phi_{k}\right|^{2} d u d v
$$

Then

$$
-T\left(D_{1}\right)=4 \iint_{D_{1}} g^{\# 2} d u d v, g
$$


the area of the image of $D_{1}$ by $g$ covering over $S$.

THEOREM 2. Let $x: D \rightarrow \mathbb{R}^{3}$ be a regular minimal surface contained in no plane. Suppose that $c \in M_{3}(K)$ exists and suppose further that the Jordan domain $\Delta$ bounded by $c$ is contained in $D$. Then,

$$
-T(\Delta)=\pi\left(Z_{\Delta}^{\prime}+P_{\Delta}-n\right)
$$

where $Z_{\Delta}^{\prime}$ is the sum of all orders of all the distinct zeros of $g^{\prime}$ in $\Delta$, while $P_{\Delta}$ is the sum of all orders of all the distinct $n$ poles of $g$ in $\Delta$.

In particular, if $g^{\#}$ never vanishes in $D$, then the right-hand side of (4) is zero. Thus, either $M_{3}(K)$ is empty or else each Jordan domain bounded by $c \in M_{3}(K)$ is not contained in $D$.

There does exist $x$ for which $\Delta \subset D$ actually happens as described in Theorem 2; see TYPE 3 in the next section.

\section{EXAMPLES}

Suppose that $D \subset \mathbb{C}$ is simply connected and $g$ is nonconstant and analytic in $D$. With the aid of $g$ we can construct a minimal surface $x: D \rightarrow \mathbb{R}^{3}$ as follows:

$$
\begin{aligned}
& x_{1}(w)=\frac{1}{2} \operatorname{Re} \int_{a}^{w}\left\{1-g(\zeta)^{2}\right\} d \zeta, \\
& x_{2}(w)=\frac{1}{2} \operatorname{Re} \int_{a}^{w} i\left\{1+g(\zeta)^{2}\right\} d \zeta, \\
& x_{3}(w)=\operatorname{Re} \int_{a}^{w} g(\zeta) d \zeta
\end{aligned}
$$

where $a$ is a fixed point of $D$. The Gauss map is just $g$; see $[2$, p.64]. Therefore,

$$
\frac{\sqrt{-K(w)}}{4}=\frac{\left|g^{\prime}(w)\right|}{\left(1+|g(w)|^{2}\right)^{2}}, w \in D .
$$

TYPE 1. Let $D=\mathbb{C}$ and $g(w)=w$. Then $M(K)=\{0\}$. (Enneper's surface)

TYPE 2. Let $D=\mathbb{C}$ and $g(w)=e^{w}$. Then $M(K)=\{\operatorname{Re} w=-(1 / 2) \log 3\}$.

TYPE 3. Let $D=\mathbb{C}$ and $g(w)=w^{2}$. Then $M(K)=\left\{|w|=7^{-1 / 4}\right\}$.

The restriction of the above surfaces to $\{|w|>1\}$ yields $M(K)=\emptyset$. A problem is to find $x: D \rightarrow \mathbb{R}^{3}$ for which two or three types appear at the same time for $M(K)$.

It would be interesting to consider the typical minimal surfaces given in the nonparametric form, namely: 
The helicoid:

$$
x_{3}=\tan ^{-1}\left(\frac{x_{2}}{x_{1}}\right), \quad\left(x_{1}, x_{2}\right) \in \mathbb{R}^{2} .
$$

The catenoid:

$$
x_{3}=\cosh ^{-1} \sqrt{x_{1}^{2}+x_{2}^{2}}, \quad x_{1}^{2}+x_{2}^{2} \geqslant 1 .
$$

See [2, pp.17-18] and [3,pp.34 and 47]. When $\left(x_{1}, x_{2}\right)=(0,0)$ in the helicoid we interpret this to express the $x_{3}$-axis.

A parametric form of the helicoid is then given by $x: \mathbb{C} \rightarrow \mathbb{R}^{3}$, where,

$$
\begin{aligned}
& x_{1}(w)=\sinh u \cos v, \\
& x_{2}(w)=\sinh u \sin v, \\
& x_{3}(w)=v .
\end{aligned}
$$

Thus, $f(w)=e^{-w}$ and $g(w)=-i e^{w}$, so that a calculation shows that $M(K)$ is the imaginary axis in $\mathbb{C}$, which corresponds to the $x_{3}$-axis lying on the surface.

A parametric form of the catenoid is given by $x: \mathbb{C} \backslash\{0\} \rightarrow \mathbb{R}^{3}$, where

$$
\begin{aligned}
& x_{1}(w)=-\frac{u}{2}\left(1+\frac{1}{|w|^{2}}\right), \\
& x_{2}(w)=\frac{v}{2}\left(1+\frac{1}{|w|^{2}}\right), \\
& x_{3}(w)=\log |w| .
\end{aligned}
$$

Thus, $f(w)=-1$ and $g(w)=-1 / w$, so that a calculation shows that $M(K)$ is the unit circle, which corresponds to the unit circle on the surface.

Note that, in all examples in this section, $K$ actually attains the global minimum at each point of $M(K)$.

\section{Proof of Theorem 1}

It suffices to prove the following proposition:

(I). Let $a \in M(K)$ be an accumulation point of $M(K)$. Then there exists $\delta>0$ such that $M(K) \cap\{|w-a|<\delta\}$ is a simple analytic arc with both terminal points on the circle $\{|w-a|=\delta\}$.

Lemma 1. Let $G$ be analytic and $H$ be meromorphic in a domain $D_{1} \subset \mathbb{C}$. Suppose that

$$
L(G, H)=\left\{w \in D_{1} ; \overline{G(w)}=H(w)\right\}
$$


has an accumulation point $a \in D_{1}$ and $G^{\prime}(a) \neq 0$. Then there exists an open disk $U(a)$ of centre $a$ such that $U(a) \cap L(G, H)$ is a simple, analytic arc passing through $a$ with both terminal points on the circle $\partial U(a)$.

The proof of this lemma is the same as that of [3, Lemma 1] (see also [4]) in case $G(w) \equiv w$. In the general case, let $V(a)$ be an open disk with centre $a$ where $G$ is univalent. Regarding $G(V(a))$ as $D_{1}, G(w)$ as $w$, and $H$ as $H \circ G^{-1}$, we can reduce this case to the case specified in the above.

We are ready to prove (I). Set

$$
\Phi(w)=\frac{\sqrt{-K(w)}}{4}, \quad w \in D
$$

We first note that $g^{\#}(a) \neq 0$ for $a \in M(K)$.

Suppose the case where $g(a) \neq \infty$ and $g^{\prime}(a) \neq 0$. Then there exists $\delta_{1}>0$ such that $g$ is analytic and univalent in $\Delta_{1}=\left\{|w-a|<\delta_{1}\right\}$ and $\Phi(w) \leqslant \Phi(a)$ for each $w \in \Delta_{1}$. Hence at each $w \in \Delta_{1} \cap M(K)$ we have

$$
\frac{\partial \Phi(w)}{\partial w} / \Phi(w)=\frac{1}{2}\left(\frac{g^{\prime \prime}(w)}{g^{\prime}(w)}-\frac{f^{\prime}(w)}{f(w)}\right)-\frac{2 \overline{g(w)} g^{\prime}(w)}{1+|g(w)|^{2}}=0 .
$$

Consequently

$$
\Delta_{1} \cap M(K) \subset L(g, H)
$$

where $L(g, H)$ is considered in $\Delta_{1}$ with

$$
H=\frac{Q}{2 g^{\prime}-Q g}, \quad Q=\frac{1}{2}\left(\frac{g^{\prime \prime}}{g^{\prime}}-\frac{f^{\prime}}{f}\right) .
$$

It follows from Lemma 1 that there exists $U(a)$ such that $L_{1}=U(a) \cap L(g, H)$ is a simple analytic arc described there. Let $L_{1}: w=w(t)$ be an analytic expression with a real parameter $t$. Then,

$$
\frac{d}{d t} \Phi(w(t))=2 \operatorname{Re}\left[\left\{\frac{\partial \Phi(w)}{\partial w}\right\}_{w=w(t)} w^{\prime}(t)\right]=0
$$

on $L_{1}$. Hence $\Phi$ is constant on $L_{1}$. Furthermore, $L_{1}=U(a) \cap M(K)$. This proves (I) for the present case.

Suppose the case where $a$ is a simple pole of $g$. Then there exists $\delta_{2}>0$ such that $G=1 / g$ is analytic and univalent in $\Delta_{2}=\left\{|w-a|<\delta_{2}\right\}$ and $\Phi(w) \leqslant \Phi(a)$ in 
$\Delta_{2}$. Consequently, at each $w \in\left(\Delta_{2} \backslash\{a\}\right) \cap M(K)$, we have

$$
\frac{\partial \Phi(w)}{\partial w} / \Phi(w)=\frac{1}{2}\left(\frac{G^{\prime \prime}(w)}{G^{\prime}(w)}-\frac{f^{\prime}(w)}{f(w)}\right)-\frac{2 \overline{G(w)} G^{\prime}(w)}{1+|G(w)|^{2}}+\frac{G^{\prime}(w)}{G(w)}=0
$$

because

$$
\Phi(w)=\frac{|G(w)|^{2} G^{\#}(w)}{|f(w)|\left(1+|G(w)|^{2}\right)}
$$

Hence

$$
\left(\Delta_{2} \backslash\{a\}\right) \cap M(K) \subset L\left(G, H_{1}\right)
$$

where $L\left(G, H_{1}\right)$ is considered in $\Delta_{2}$ with

$$
H_{1}=\frac{Q_{1}}{2 G^{\prime}-Q_{1} G}, \quad Q_{1}=\frac{1}{2}\left(\frac{G^{\prime \prime}}{G^{\prime}}-\frac{f^{\prime}}{f}\right)+\frac{G^{\prime}}{G} .
$$

Thus, $a$ is an accumulation point of $L\left(G, H_{1}\right)$ and $G^{\prime}(a) \neq 0$. It follows from Lemma 1 that there exists $U(a)$ such that

$$
L_{2}=U(a) \cap L\left(G, H_{1}\right)
$$

is a simple analytic arc described there. On the other hand, $\Phi$ is constant on $L_{2} \backslash\{a\}$, so that $\Phi(w) \equiv \Phi(a), w \in L_{2}$, by the continuity of $\Phi$ at $a$. Accordingly

$$
L_{2}=U(a) \cap M(K)
$$

and this completes the proof of (I).

REMARK. We let $M^{*}(K)$ be the set of points $w_{0} \in D$ where $K$ has the (global) minimum: $K\left(w_{0}\right) \leqslant K(w), w \in D$. Suppose that $a \in D$ is an accumulation point of $M^{*}(K)(\subset M(K))$. Then there exists $c \in M_{2}(K) \cup M_{3}(K)$ such that $a \in c$. Since $K$ is constant on $c$, it follows that $c \subset M^{*}(K)$. Hence we have the analogous classification: $M_{k}^{*}(K), k=1,2,3$, of components of $M^{*}(K)$.

\section{Proof of TheOREM 2}

First of all $g^{\#}$ never vanishes on $c=\partial \Delta$ because this is the case at each point of $M(K)$. Let $\alpha_{k}, 1 \leqslant k \leqslant p$, be all the simple poles of $g$ on $c$, and let $\gamma_{k}$ be all the distinct poles of $g$ in $\Delta$ of orders $\nu_{k}, 1 \leqslant k \leqslant n$, so that

$$
P_{\Delta}=\sum_{k=1}^{n} \nu_{k}
$$


Set $A=\left\{\alpha_{1}, \ldots, \alpha_{p}, \gamma_{1}, \ldots, \gamma_{n}\right\}$. For $\varepsilon>0$ and $\alpha \in A$ we set

$$
\begin{aligned}
& \delta(\alpha, \varepsilon)=\{z ;|z-\alpha| \leqslant \varepsilon\} \\
& c(\alpha, \varepsilon)=\{z \in \Delta ;|z-\alpha|=\varepsilon\} .
\end{aligned}
$$

Then, from sufficiently small $\varepsilon$ on,

$$
\Delta(\varepsilon)=\Delta \backslash \bigcup_{\alpha \in A} \delta(\alpha, \varepsilon)
$$

is a domain bounded by Jordan curves. Set

$$
\lambda=\frac{\bar{g} g^{\prime}}{1+|g|^{2}} \text { and } \mu=i \lambda \text {. }
$$

Then the Green formula

$$
\iint_{\Delta(e)}\left(\mu_{u}-\lambda_{v}\right) d u d v=\int_{\partial \Delta(e)}(\lambda d u+\mu d v)
$$

can be rewritten as

$$
4 \iint_{\Delta(e)} g^{\#}(w)^{2} d u d v=-2 i \int_{\partial \Delta(e)} \lambda(w) d w
$$

where the line integral is in the positive sense with respect to $\Delta(\varepsilon)$.

Now, the Laurent expansion of $g$ about $\alpha \in A$ yields

$$
g(w)=(w-\alpha)^{-N} h(w) \text { in } \delta(\alpha, \varepsilon) \backslash\{\alpha\}
$$

where $h$ is analytic and zero-free in $\delta(\alpha, \varepsilon)$ and $N=1$ if $\alpha=\alpha_{k}$, while $N=\nu_{k}$ if $\alpha=\gamma_{k}$. The differentiation yields that

$$
g^{\prime}(w)=(w-\alpha)^{-N-1} \Psi(w) \text { in } \delta(\alpha, \varepsilon) \backslash\{\alpha\}
$$

where

$$
\Psi(w)=-N h(w)+(w-\alpha) h^{\prime}(w)
$$

Since

$$
\varepsilon e^{i t} \lambda\left(\varepsilon e^{i t}+\alpha\right)=\frac{\overline{h\left(\varepsilon e^{i t}+\alpha\right)} \Psi\left(\varepsilon e^{i t}+\alpha\right)}{\varepsilon^{2 N}+\left|h\left(\varepsilon e^{i t}+\alpha\right)\right|^{2}} \rightarrow-N \text { as } \varepsilon \rightarrow 0
$$

uniformly for real $t$, it follows that

$$
\int_{c(\alpha, e)} \lambda(w) d w \rightarrow \begin{cases}\pi i & \text { if } \alpha=\alpha_{k} \\ 2 \pi \nu_{k} i & \text { if } \alpha=\gamma_{k}\end{cases}
$$


as $\varepsilon \rightarrow 0$, where the integral is in the clockwise sense. Letting $\varepsilon \rightarrow 0$ in (4.1), we now have

$$
\begin{aligned}
4 \iint_{\Delta} g^{\#}(w)^{2} d u d v & =-2 i \int_{c} \lambda(w) d w+2 \pi p+4 \pi P_{\Delta} \\
& =\frac{1}{2 i} \int_{c}\left(\frac{g^{\prime \prime}(w)}{g^{\prime}(w)}-\frac{f^{\prime}(w)}{f(w)}\right) d w+2 \pi p+4 \pi P_{\Delta}
\end{aligned}
$$

by $\partial \Phi(w) / \partial w=0$ on $c$.

We remember that $f$ vanishes precisely at the poles of $g$. Thus, $\gamma$ is a zero of order $2 \nu$ of $f$ if and only if $\gamma$ is a pole of order $\nu$ of $g$. Hence,

$$
\frac{1}{2 \pi i} \int_{\theta \Delta_{0}(\varepsilon)}\left(\frac{g^{\prime \prime}(w)}{g^{\prime}(w)}-\frac{f^{\prime}(w)}{f(w)}\right) d w=Z_{\Delta}^{\prime}-\left(3 P_{\Delta}+n\right)
$$

where

$$
\Delta_{0}(\varepsilon)=\Delta \backslash \bigcup_{k=1}^{p} \delta\left(\alpha_{k}, \varepsilon\right)
$$

We have in $\delta(\alpha, \varepsilon) \backslash\{\alpha\}, \alpha=\alpha_{k}$,

$$
\frac{g^{\prime \prime}(w)}{g^{\prime}(w)}-\frac{f^{\prime}(w)}{f(w)}=\frac{-4}{w-\alpha}+\frac{X^{\prime}(w)}{X(w)}
$$

where $X$ is analytic and zero-free in $\delta(\alpha, \varepsilon)$ for small $\varepsilon>0$. Consequently, letting $\varepsilon \rightarrow 0$ in the left-hand side of (4.4) we have the identity

$$
\frac{1}{2 \pi i} \int_{c}\left(\frac{g^{\prime \prime}(w)}{g^{\prime}(w)}-\frac{f^{\prime}(w)}{f(w)}\right) d w+2 p=Z_{\Delta}^{\prime}-3 P_{\Delta}-n
$$

Combining (4.3) and (4.5) we have (4).

\section{REFERENCES}

[1] J.C.C. Nitsche, Vorlesungen über Minimalflächen (Springer-Verlag, Berlin, Heidelberg, New York, 1975).

[2] R. Osserman, A survey of minimal surfaces (Van Nostrand Reinhold Company, New York, 1969).

[3] S. Ruscheweyh and K.-J. Wirths, 'On extreme Bloch functions with prescribed critical points', Math. Z. 180 (1982), 91-105.

[4] S. Ruscheweyh and K.-J. Wirths, 'Extreme Bloch functions with many critical points', Analysis 4 (1984), 237-247.

Department of Mathematics

Tokyo Metropolitan University

Minami Osawa, Hachioji

Tokyo 192-03

Japan 